\title{
Panniculitis: a report of four cases and literature review
}

\author{
S M Randle, M B Richter, R G Palmer, A Price, B M Ansell
}

\begin{abstract}
Panniculitis is a disease with many causes and associations. The classification of clinical subtypes is unsatisfactory and hampered by the use of eponyms.

Four children with recurring panniculitis are described and their histology presented. Three had subcutaneous fat atrophy with lobular panniculitis on biopsy; all responded well to corticosteroids. The fourth child had a septal panniculitis with no atrophy of subcutaneous tissues and only a partial response to treatment with corticosteroids.

A widely accepted precise histological classification of panniculitis is needed to enable accurate predictions of the outcome of this serious disorder.
\end{abstract}

Panniculitis is an uncommon but fascinating condition in childhood. Inflammation of the fat may arise spontaneously or occur as a feature of distinct illnesses such as pancreatitis, ${ }^{1}$ bacterial septicaemia, ${ }^{2}$ systemic lupus erythematosis, ${ }^{3}$ polyarteritis, ${ }^{1}$ and malignancy. ${ }^{4}$ The literature abounds with isolated case reports using eponyms such as 'Weber-Christian syndrome's and 'Rothman-Makai panniculitis'. ${ }^{6}$ Although attempts have been made to classify histological subtypes of panniculitis, ${ }^{17}$ no universally recognised classification is in use to aid retrospective studies. The various forms of lipodystrophy are often included in the classification of panniculitis, ${ }^{8}$ which then becomes further confused.

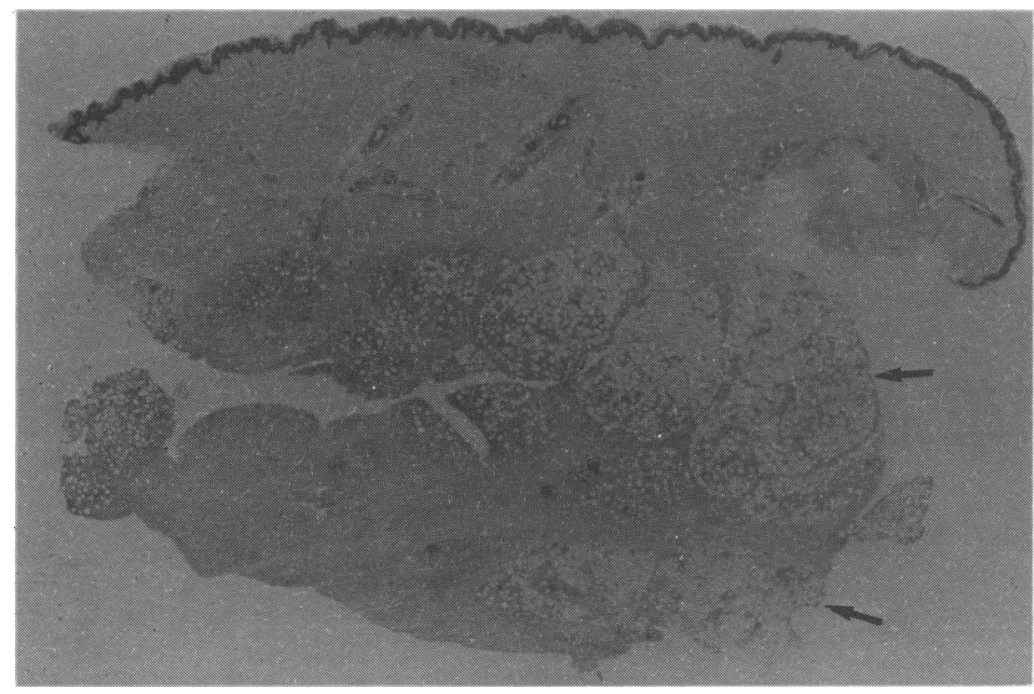

Figure 1 Case 1: histological appearance of lobular panniculitis (arrows). (Haematoxylin and eosin stain $\times 10$.)
We report four cases of spontaneously occurring panniculitis, one in an infant and three in older children. Follow up of our four children over several years suggests that lobular panniculitis is associated with significant fat atrophy at the site of previously active lesions and a good response to corticosteroids. Septal panniculitis, however, has no associated atrophy of fat and only a partial response to corticosteroids.

\section{Case reports}

CASE 1

A 6 year old girl first presented in 1975 with an upper respiratory infection and painful lumps on both thighs. Both problems resolved spontaneously. The painful lumps reappeared three years later and biopsy specimen of a nodule demonstrated lobular panniculitis (fig 1).

Other investigations included a haemoglobin concentration of $94 \mathrm{~g} / \mathrm{l}$, total white cell count of $11.9 \times 10^{9} / 1$, erythrocyte sedimentation rate (ESR) of $60 \mathrm{~mm} /$ hour, a significant rise in antistreptolysin 0 titre (ASOT) over two months, a positive rheumatoid factor, and raised immune complexes. Serum C3 concentration was $1.65 \mathrm{~g} / \mathrm{l}$ (normal range $0 \cdot 8-1 \cdot 70 \mathrm{~g} / \mathrm{l}$ ) and C4 $0.23 \mathrm{~g} / \mathrm{l}$ (normal range $0.15-0.45 \mathrm{~g} / \mathrm{l}$ ). Serum IgG was $28 \mathrm{~g} / \mathrm{l}$ (normal range 6.5-16.5 g/l). Direct Coombs test, the Venereal Disease Research Laboratory test, Treponema pallidum haemagglutination assay, and tests for antinuclear and anti-DNA antibodies gave negative results. No cryoglobulins or cold agglutinins were detected, and serum $\operatorname{IgA}, \operatorname{IgM}$, euglobulin lysis and fibrin plate lysis. were normal.

Prednisolone was given in a dose of 30 $\mathrm{mg} /$ day orally from January 1979 tapering to 30 $\mathrm{mg}$ and $5 \mathrm{mg}$ on alternate days by February 1979. The prednisolone dose was steadily reduced over the next five months, ceasing in July 1979 at which time there was resolution of the lesions with appreciable loss of subcutaneous fat at the sites of the previous nodules. The nodules reappeared three months after cessation of treatment. Treatment with prednisolone was again successful in controlling the symptoms when given in a dose of $30 \mathrm{mg} / \mathrm{day}$ orally, initially, and decreased steadily as before over the next nine months until ceasing in July 1982. Currently the child remains well and free of new lesions.

CASE 2

This girl was completely normal until the age of 5 months when she developed red hot lumps on both legs. By 16 months of age the lesions had 
spread to involve her face, arms, and trunk. There were no systemic symptoms. A skin biopsy specimen showed a septal panniculitis (fig 2) that responded temporarily to prednisolone in small doses.

At 3.5 years of age she developed fever, joint pains, and stiffness with increasing numbers of nodules appearing after bouts of tonsillitis. A trial of prophylactic penicillin decreased the number of infections but did not appear to modify the disease process.

Subsequent treatment included chloroquine, indomethacin, naproxen, and sulphasalazine; none of these drugs resulted in any lasting benefit. Prednisolone was used in small doses (for example, $5 \mathrm{mg}$ ) daily until the age of 5.5 years when alternate day treatment was begun up to a dose of $20 \mathrm{mg}$ at 11 years old. Increases

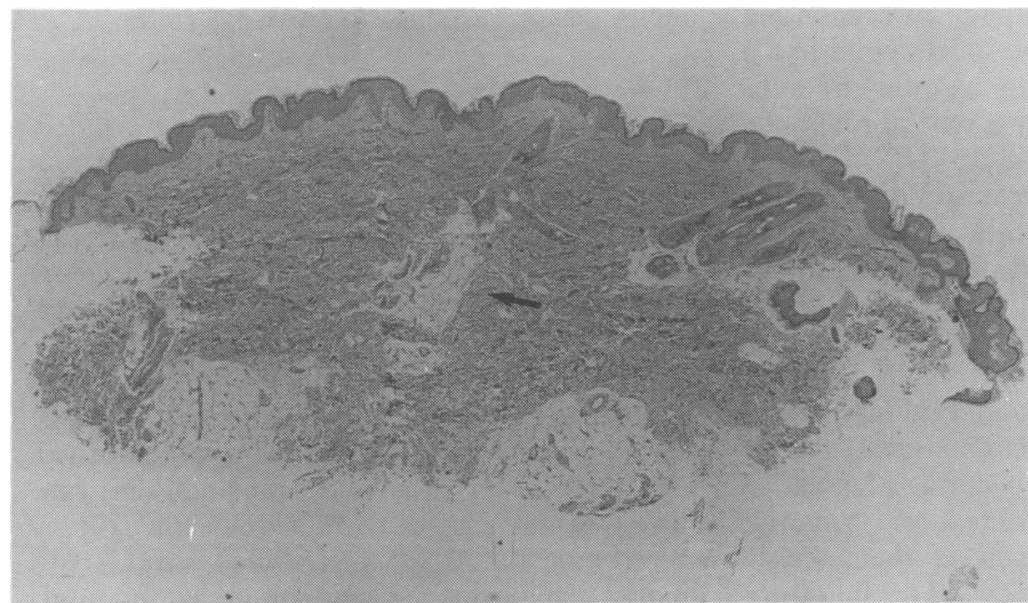

Figure 2 In contrast to fig 1 this biopsy shows relatively normal fat lobules with inflammation of the surrounding septa (arrow). (Haematoxylin and eosin stain $\times 10$.)

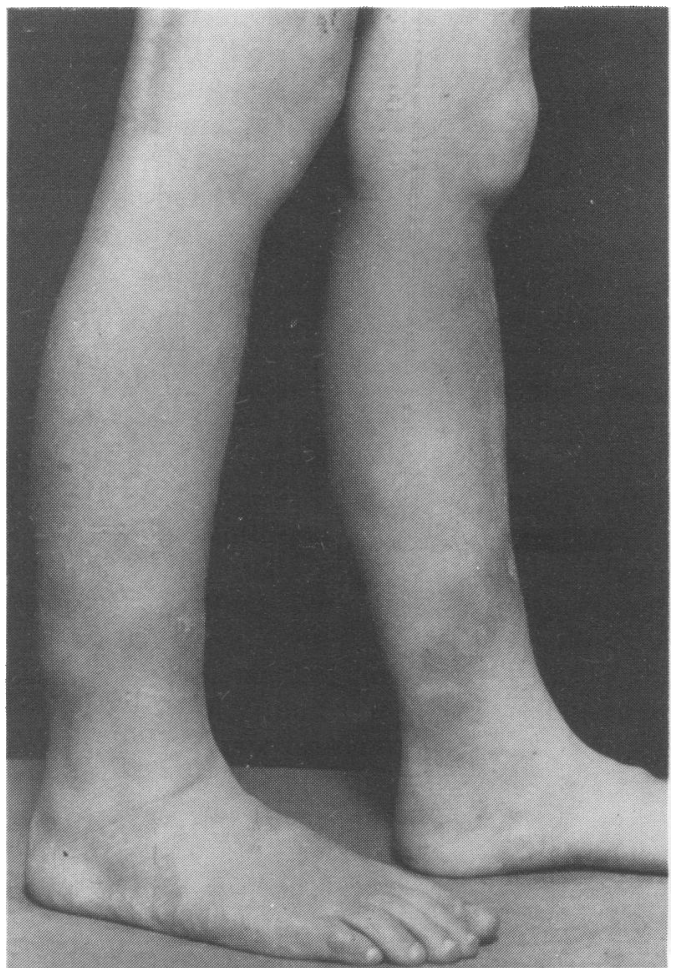

Figure 3 Case 2: calf nodules of septal panniculitis in relapse. in prednisolone dose led to partial improvements but relapses still occurred (fig 3). Temporary improvement was seen after three pulses of intravenous methylprednisolone were given in response to an acute exacerbation of the disease. Dapsone was started at 13 years of age with some improvement of fevers and nodules.

During the course of this girl's illness her haemoglobin has remained at $100-111 \mathrm{~g} / 1$ and her ESR has ranged from 13 to $30 \mathrm{~mm} /$ hour.

Tests for antinuclear antibody, immune complexes, autoantibodies to gastric parietal cells, smooth muscle, mitochondria, reticulin and thyroglobulin gave negative results. ASOT, throat swab, and urine and blood cultures were also negative. Plasma urea, electrolytes, C reactive protein, triglycerides, cholesterol, red cell folate, serum complement, immunoglobulins, and B12 concentrations and bone marrow examination were all normal.

CASE 3

In December 1985 an 11 year old girl developed painful erythematous indurated lesions over her feet and legs (fig 4) after recovering from a sore throat. She was not febrile but appeared lethargic. At the same time the metatarsophalangeal joints of both feet were tender.

There was no improvement with penicillin, aspirin, other non-steroidal anti-inflammatory drugs, and bed rest. Her haemoglobin concentration was $113 \mathrm{~g} / 1$, white cell count $6.8 \times 10^{9} / 1$, and ESR $60 \mathrm{~mm} /$ hour. Rheumatoid factor was positive (RAHA 1:1280) initially, becoming negative by November 1987 . Plasma $C$ reactive protein was $27000 \mu \mathrm{g} / 1$ (normal <8000). The

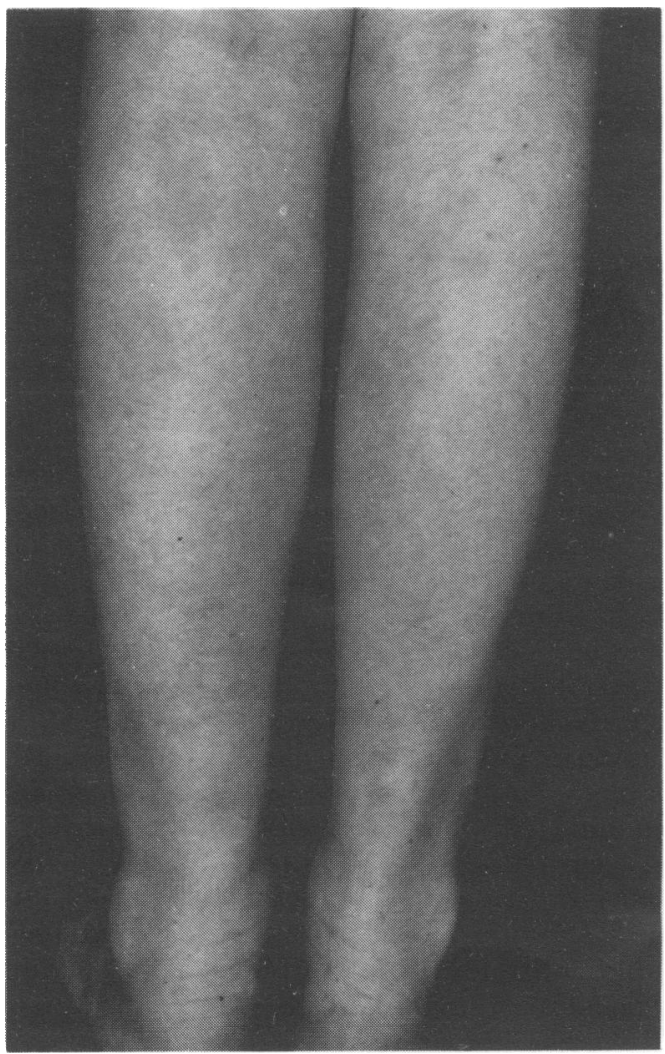

Figure 4 Case 3: swelling and erythema of the legs in the acute phase of lobular panniculitis. 
plasma urea and electrolytes, liver function tests, amylase activity, ASOT, serum immunoglobulins and complement, urine analysis, nose and throat swabs, and chest radiography all gave normal results. Autoantibodies to parietal cells, mitochondria, smooth muscle, reticulin, thyroid microsomes, and thyroglobulin were not detected. Antinuclear and anti-DNA antibodies were negative and antibodies to extractable nuclear antigen were not detected.

One month after onset of the illness nodules began to appear on the child's arms. A skin biopsy specimen demonstrated lobular panniculitis and prednisolone was started at a dose of $40 \mathrm{mg}$ alternating with $10 \mathrm{mg}$ daily. She became more energetic and the skin lesions gradually resolved, leaving large areas of subcutaneous fat loss (fig 5). Prednisolone intake was cautiously reduced as three small lesions persisted and occasional lesions appeared at sites of minor skin trauma. By August 1988 the prednisolone dose was $25 \mathrm{mg}$ on alternate days, the child felt well, and the ESR had fallen to $6 \mathrm{~mm} / \mathrm{hour}$.

CASE 4

A girl weighing $4500 \mathrm{~g}$ was born by elective caesarean section after a normal pregnancy in August 1985. She had signs of ventricular and atrial septal defects leading to congestive cardiac failure by 5 weeks of age. Corrective cardiac surgery was performed three weeks later after medical treatment had failed to control her condition.

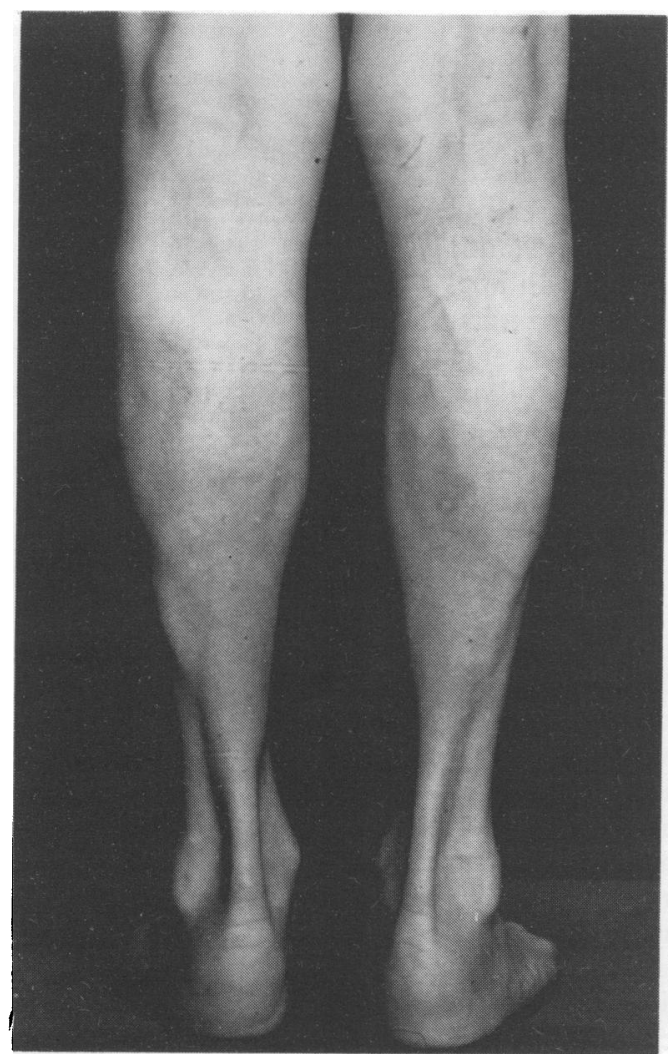

Figure 5 Case 3: appreciable subcutaneous fat loss seent after resolution of the acute inflammatory stage of lobular panniculitis.
Postoperatively the baby required intensive respiratory and cardiovascular support but was able to go home after two weeks in hospital. Shortly afterwards she developed red tender lumps on her trunk, thighs, arms, and scalp. These lumps resolved spontaneously but reappeared five weeks later on the baby's arms, shoulder, and face where they were sufficiently tender to interfere with feeding.

A biopsy specimen of an active lesion showed lobular panniculitis that responded well to prednisolone in a dose of $1.5 \mathrm{mg} / \mathrm{kg} /$ day. The dose of steroid was reduced over the next five months and there has been no recurrence of disease off treatment.

Investigations at the time of biopsy showed a haemoglobin concentration of $95 \mathrm{~g} / \mathrm{l}$, white cell count of $12 \cdot 2 \times 10^{9} / 1$, and an ESR of $13 \mathrm{~mm} /$ hour. ESR at presentation was $40 \mathrm{~mm} /$ hour. Serum amylase and creatine phosphokinase activities and ASOT were normal. No immune complexes were detected.

\section{Discussion}

Our four cases demonstrate the two histological types of panniculitis previously described, lobular and septal. ${ }^{1}$ Although these types have been recognised in the past no attempt has been made to correlate histological type with response to treatment. Our review of the literature supports this paper's finding that lobular panniculitis responds better to treatment with prednisolone than the septal type.

Two of our children were infants at the onset of the illness. Most of the infants described previously have had significant systemic symptoms and in some cases a fatal outcome. ${ }^{6}$ Our infant with lobular panniculitis has done well on steroids alone. She now has no fat atrophy and is off treatment with no recurrences. Taylor ${ }^{9}$ and more recently Conway et $a^{10}$ have also described cases of panniculitis with onset in infancy where the clinical course has been relatively mild and there has been a good response to treatment. Our child with septal panniculitis whose disease began at 5 months of age, although still having problems, has not succumbed and has not developed symptoms of perivisceral disease.

The nature of the inflammatory infiltrate found on histology varies according to the disease stage at which the biopsy is performed. ${ }^{11}$ It is important to obtain adequate biopsy specimens early in the disease as once the end stage is reached, subcutaneous fibrosis of the panniculis may be all that is seen and the lobular architecture of the fat is distorted. Punch biopsies are not recommended because they may not obtain adequate samples of fatty tissue because of inflammation of the subcutaneous tissue, which leads to decreased adherence of the dermis to the fat in the early stages. ${ }^{1}$

Unless an acute lesion is biopsied histology can be meaningless so that children presenting with a loss of subcutaneous fat are particularly difficult to diagnose. In the four patients described by Peters and Winkelmann nodules were not present at the beginning of the disease 
or if present were obvious only briefly. ${ }^{12}$ Three of their patients were thought to have lipodystrophy until biopsies showed panniculitis.

Upper respiratory tract infections preceded the panniculitis in two of our cases with lobular panniculitis. The child with septal panniculitis suffered frequent exacerbations of her disease after bouts of tonsillitis as did a child with lobular panniculitis described by Gupta and Rasmussen. ${ }^{13}$ Septal panniculitis has been recently associated with Lyme disease. ${ }^{14}$ There is a similarity between this form of panniculitis and erythema nodosum. Erythema nodosum is considered to be a hypersensitivity reaction to a variety of antigenic stimuli and is seen during several infectious diseases including those caused by $\beta$ haemolytic streptococci. It is clear from the literature that the presence of infection is not predictive of the histological type of panniculitis nor the clinical outcome.

The infant who underwent corrective cardiac surgery was initially thought to have 'cold panniculitis' resulting from the hypothermia during surgery. A recent case report describes an infant with similar problems after cardiac surgery and the diagnosis of subcutaneous fat necrosis of the newborn was made on the histological findings. ${ }^{15}$ Cold panniculitis has previously been described but is localised to the area affected by the hypothermia, begins hours to at most a few days after the insult, and does not spontaneously recur without further exposure to cold. ${ }^{16}$ Our child's lesions recurred too long after cardiac surgery to be consistent with cold panniculitis. Nor is this child's condition compatible clinically or histologically with sclerema neonatorum or subcutaneous fat necrosis of the newborn.

Immunological abnormalities have previously been reported in association with panniculitis. Allen-Mersh described a 7 year old girl who first presented with a panniculitis of the foot and who then went on to develop diabetes mellitus, hepatic cirrhosis, red cell surface antibodies, and antibodies to mitochondria and gastric parietal cells. ${ }^{17}$ Recently Billings et al reported three cases of lobular panniculitis. ${ }^{18}$ One of the patients had insulin dependent diabetes and Hashimoto's thyroiditis, the second later developed juvenile chronic arthritis, and the third insulin dependent diabetes.

Hendricks et al describe a child whose disease began after immunisation and another who did not develop new lesions while he was lymphocyte depleted. ${ }^{6}$

Our two older children with lobular panniculitis had positive rheumatoid factors. One had a raised ASOT and immune complexes. All children had negative autoantibody screens. The significance of these isolated findings is not clear and a review of the literature does not suggest any correlation between the nature of the immunological disturbance and the histological type of panniculitis.

It is clear that many factors are involved in the initiation of panniculitis. It is possible that various physical and chemical agents and infections expose parts of the lipocyte increasing its vulnerability to attack by autoimmune mechanisms. This is supported by the increased incidence of panniculitis among children with $\alpha_{1}$-antitrypsin deficiency. ${ }^{19}$

Steroids have been the mainstay of treatment of panniculitis but some children do not respond. ${ }^{20}$ Other agents tried with success have been chloroquine, ${ }^{2111}$ azathioprine, ${ }^{10}$ cyclophosphamide, ${ }^{22}$ and more recently dapsone. ${ }^{23} 24$

Panniculitis is a disease with at least two distinct histological subtypes. Current treatments are often based on the results presented in numerous case reports rather than the outcome of prospective trials of treatment. The incidence of panniculitis is low but for a particular child this illness may cause serious disability or even death. It is for these reasons it is suggested that all children who present with recurrent painful nodules of panniculitis have an early and adequate biopsy to ascertain the histology. Prompt treatment may prevent further severe illness as well as disfiguring subcutaneous fat loss.

1 Ackerman B. Panniculitis. Histologic diagnosis of inflammatory skin diseases. Philadelphia: Lea and Febiger, 1978:779-825.

2 Bagel J, Grossman ME. Subcutaneous nodules in pseudomonas sepsis. Am $\mathcal{F}$ Med 1986;80:528-9.

monas sepsis. Am 7 Med $1986 ; 80: 528-9$.
3 inkelmann RK. Panniculitis in connective tissue disease. Arch Dermatol 1983;119:336-44

4 Aronson IK, West DP, Vanakojis D, Ronan SG, Iossifides I, Zeitz HJ. Panniculitis associated with cutaneous T-cell lymphoma and cytophagocytic histiocytosis. $\mathrm{Br} \mathcal{F}$ Dermatol 1985;112:87-96.

5 Edge J, Dunger DB, Dillon MJ. Weber-Christian panniculitis and chronic active hepatitis. Eur $\mathcal{F}$ Pediatr 1986;145:227-9.

6 Hendricks WM, Ahmad M, Gratz E. Weber-Christian syndrome in infancy. $\mathrm{Br} \mathcal{F}$ Dermatol 1978;98:175-86.

7 Niemi KM, Forstrom L, Hannuksela M, Mustakollio KK, Salo OP. Nodules on the legs. A clinical, histological and immunohistological study of 82 patients representing different types of nodular panniculitis. Acta Derm Venereol (Stockh) 1977;57:145-54.

8 Patterson JW. Panniculitis. Acta Dermatol 1987;123:1615-8.

9 Taylor GA. Prolonged remission of Weber-Christian syndrome in an infant. Clin Pediatr (Phila) 1981;20:521-3.

10 Conway SP, Smithells RW, Peters WM. Weber-Christian panniculitis. Ann Rheum Dis 1987;46:339-41.

11 Aronson IK,Zeitz HJ, Variakoiis D. Panniculitis in childhood. Pediatr Dermatol 1988;5:216-30.

12 Peters MS, Winkelmann RK. Localised lipoatrophy (atrophic connective tissue disease panniculitis). Arch Dermatol 1980;116:1363-8.

13 Gupta AK, Rasmussen JE. Multiple areas of localized tissue loss in a child. Arch Dermatol 1986;122:1199-2000.

14 Kramer N, Rickert RR, Brodkin RH, Rosenstein EO. Septal panniculitis as a manifestation of Lyme disease. Am $\mathcal{F}$ Med 1986;81:149-52.

15 Silverman AK, Mickels EH, Rasmussen JE. Subcutaneous fat necrosis in an infant occurring after hypothermic cardiac surgery. I Am Acad Dermatol 1986;15:331-6.

16 Hultcrantz E. Haxthausen's disease. Cold panniculitis in children. F Laryngol Otol 1986;100:1329-32.

17 Allen-Mersh TG. Weber-Christian panniculitis and autoimmune disease: a case report. F Clin Pathol 1976;29:144-9.

immune disease: a case report. F Clin Pathol 1976;29:144-9.
18 Billings JK, Milgraum SS, Gupta AK, Headington JT, Rasmussen JE. Lipoatrophic panniculitis: a possible Rasmussen JE. Lipoatrophic panniculitis: a possible
autoimmune inflammatory disease of fat. Arch Dermatol autoimmune inflam

19 Rubenstein HM, Jaffer AM, Kuduna JC, Lortratanakul Y. Alpha 1-antitryspin deficiency with severe panniculitis. Report of two cases. Ann Intern Med 1977;86:742-4.

20 Winkelmann RK, McEvoy MT, Peters MS. Lipophagic panniculitis of childhood. $\mathcal{J}$ Am Acad Dermatol 1989;21: 971-8.

21 Shelley WB. Chloroquine-induced remission of nodular panniculitis present for 15 years. $\mathcal{F}$ Am Acad Dermatol 1981;5:168-70.

22 Kirch W, Duhrsen V, Hoensel H, Ohnhaus E. Cyclophosphamide-induced remission in Weber-Christian panniculitis. Rheumatol Int 1985;5:239-40.

23 Uplekar MW, Antia NH. Dapsone dependent nodular panniculitis. Indian $\mathcal{Y}$ Lepr 1986;58:286-90.

24 Roth DE, Schikler KS, Callen JP. Annular atrophic connective tissue panniculitis of the ankles. 7 Am Acad Dermato 1989;21:1152-6. 